\title{
Acceleration of a Domain Decomposition Method for Advection-Diffusion Problems
}

Gert Lube $^{1}$, Tobias Knopp ${ }^{2}$, and Gerd Rapin ${ }^{2}$

1 University of Göttingen, Institute of Numerical and Applied Mathematics (http://www.num.math.uni-goettingen.de/lube/)

${ }^{2}$ University of Göttingen, Institute of Numerical and Applied Mathematics

Summary. For advection-diffusion problems we show that a non-overlapping domain decomposition method with interface conditions of Robin type can be accelerated by using a critical parameter of the transmission condition in a cyclic way.

\section{Introduction}

We consider a non-overlapping domain decomposition method (DDM) with Robin transmission conditions for the advection-diffusion-reaction model

$$
\begin{aligned}
L u:=-\epsilon \Delta u+(\mathbf{b} \cdot \nabla) u+c u & =f & & \text { in } \Omega \\
u & =0 & & \text { on } \partial \Omega
\end{aligned}
$$

in a bounded polyhedral domain $\Omega \subset \mathbf{R}^{d}$ with a Lipschitz boundary $\partial \Omega$ and $0<\epsilon \leq 1, \mathbf{b} \in\left[H^{1}(\Omega) \cap L^{\infty}(\Omega)\right]^{d}, c \in L^{\infty}(\Omega), f \in L^{2}(\Omega), c-\frac{1}{2} \nabla \cdot \mathbf{b} \geq 0$.

Let $\left\{\Omega_{k}\right\}$ be a non-overlapping macro partition with $\bar{\Omega}=\cup_{k=1}^{N} \bar{\Omega}_{k}$. The goal of the well-known DDM of Robin type (Lions [1990]) is to enforce (in appropriate trace spaces) continuity of the solution $u$ and of the diffusive and advective fluxes $\epsilon \nabla u \cdot \mathbf{n}_{k j}$ resp. $-\frac{1}{2}\left(\mathbf{b} \cdot \mathbf{n}_{k j}\right) u$ on the interfaces $\Gamma_{k j}:=\partial \Omega_{k} \cap \partial \Omega_{j}$. The algorithm reads in strong form:

For given $u_{k}^{n}, n \in \mathbf{N}_{0}$, in each $\Omega_{k}$, find (in parallel) $u_{k}^{n+1}$, such that

$$
\begin{aligned}
L u_{k}^{n+1} & =f & & \text { in } \Omega_{k} \\
u_{k}^{n+1} & =0 & & \text { on } \partial \Omega_{k} \cap \partial \Omega \\
\Phi_{k}\left(u_{k}^{n+1}\right) & =\Phi_{k}\left(u_{j}^{n}\right) & & \text { on } \Gamma_{k j}
\end{aligned}
$$

with $\Phi_{k}(u)=\epsilon \boldsymbol{\nabla} u \cdot \mathbf{n}_{k j}+\left(z_{k}-\frac{1}{2} \mathbf{b} \cdot \mathbf{n}_{k j}\right) u$ on $\Gamma_{k j}, k \neq j$ and the outer normal vector $\mathbf{n}_{k j}$ on $\partial \Omega_{k} \cap \partial \Omega_{j}$. By determining the interface parameter $z_{k}>0$ in a proper way the convergence of the method (3)-(5) can be accelerated.

Let $\mathcal{T}_{h}$ be an admissible, quasi-uniform triangulation of $\Omega$ being aligned with the macro partition. $V_{h}:=\left\{v \in H_{0}^{1}(\Omega)|v|_{K} \in \mathcal{P}_{l}(K) \forall K \in \mathcal{T}_{h}\right\}$ denotes a conforming finite element (FE) space of order $l$. The stabilized FE method 


$$
\begin{gathered}
\text { Find } u_{h} \in V_{h}, \text { such that : } \quad a^{s}\left(u_{h}, v_{h}\right)=l^{s}\left(v_{h}\right) \quad \forall v_{h} \in V_{h}, \\
a^{s}(u, v)=(\epsilon \boldsymbol{\nabla} u, \nabla v)_{\Omega}+(\mathbf{b} \cdot \nabla u+c u, v)_{\Omega}+\sum_{T \in \mathcal{T}_{h}}\left(\delta_{T} L u, \mathbf{b} \cdot \nabla v\right)_{T}, \\
l^{s}(v)=(f, v)_{\Omega}+\sum_{T \in \mathcal{T}_{h}}\left(\delta_{T} f, \mathbf{b} \cdot \nabla v\right)_{T}, \quad \delta_{T} \sim h_{T}^{2}\left(\epsilon+h_{T}\|\mathbf{b}\|_{\infty, T}\right)^{-1}
\end{gathered}
$$

with residual stabilization provides improved stability and accuracy (w.r.t. the streamline derivative $\mathbf{b} \cdot \nabla u$ ) in the hyperbolic limit $\epsilon \rightarrow 0$ of (1)-(2).

Assume, for simplicity, that the macro partition has no interior crosspoints. Then we restrict the bilinear and linear forms $a^{s}$ and $l^{s}$ to each subdomain by $a_{k}^{s}=\left.a^{s}\right|_{\Omega_{k}}$ and $l_{k}^{s}=\left.l^{s}\right|_{\Omega_{k}}$. Moreover, we define $V_{k, h}=\left.V_{h}\right|_{\Omega_{k}}$ and the trace space $W_{k j, h}=\left.V_{h}\right|_{\Gamma_{k j}}$. Finally, we denote by $\langle\cdot, \cdot\rangle_{\Gamma_{k j}}$ the dual product on $\left(W_{k j, h}\right)^{*} \times W_{k j, h}$. Then the weak formulation of the fully discretized DDM is given by

(I) Parallel computation step :

For $k=1, \ldots, N$ find $u_{h, k}^{n+1} \in V_{k, h}$ such that for all $v_{k} \in V_{k, h}$ :

$$
a_{k}^{s}\left(u_{h, k}^{n+1}, v_{k}\right)+\left\langle z_{k} u_{h, k}^{n+1}, v_{k}\right\rangle_{\Gamma_{k}}=l_{k}^{s}\left(v_{k}\right)+\sum_{j(\neq k)}\left\langle\Lambda_{j k}^{n}, v_{k}\right\rangle_{\Gamma_{k j}} .
$$

(II) Communication step :

For all $k \neq j$, update Lagrange multipliers $\Lambda_{k j}^{n+1} \in W_{k j, h}^{*}$ such that:

$$
\left\langle\Lambda_{k j}^{n+1}, \phi\right\rangle_{\Gamma_{k j}}=\left\langle\left(z_{k}+z_{j}\right) u_{h, k}^{n+1}-\Lambda_{j k}^{n}, \phi\right\rangle_{\Gamma_{k j}}, \quad \forall \phi \in W_{k j, h} .
$$

This method is very easy to implement. It is used as a building block in a parallelized flow solver for the thermally driven incompressible Navier-Stokes problem, cf. Knopp et al. [2002]. A fast convergence of the DDM is desirable, in particular for time-dependent problems.

It is well-known that the algorithm (7)-(8) is well-posed if $z_{k}=z_{j}>0$. Moreover, the sequence $\left\{u_{k}^{n}\right\}_{n \in \mathbf{N}}$ is strongly convergent according to

$$
\left.\lim _{n \rightarrow \infty}\left\|\left|u_{h, k}^{n}-u_{h}\right|_{\Omega_{k}}\right\|\right|_{\Omega_{k}}=0
$$

where $\left\||v \||_{\Omega_{k}}:=\sqrt{a_{k}^{s}(v, v)}\right.$, Lube et al. [2000]. The convergence speed depends in a sensitive way on the parameters $z_{k}$ which have to be optimized. In Sec. 2 we review an a priori optimization introduced by Nataf and Gander. Sec. 3 is devoted to an a posteriori based approach.

\section{A-priori optimization of the interface condition}

The convergence of the Robin DDM (3)-(5) can be described in simple cases using a Fourier analysis. Nataf and Gander proposed a semi-continuous a priori optimization of the interface parameter $z$ over a relevant range $S=\left(s_{\min }, s_{\max }\right)$ of Fourier modes. An optimization is important for highly 
oscillatory solutions, e.g. for the Helmholtz equation (1)-(2) with $\mathbf{b} \equiv \mathbf{0}$ and $c / \epsilon \ll-1$. An improved idea is a cyclic change of $z$ for appropriate frequency ranges. For the symmetric case with $\mathbf{b}=\mathbf{0}$ and $\epsilon=1$, Gander and Golub [2002] proposed the following variant of the DDM (3)-(5) in $\Omega=\mathbf{R}^{2}, \Omega_{1}=\mathbf{R}^{+} \times \mathbf{R}, \Omega_{2}=\mathbf{R}^{-} \times \mathbf{R}$ with the cyclic Robin condition

$$
\boldsymbol{\nabla} u_{1}^{n+1} \cdot \mathbf{n}_{1}+z^{n \bmod (m)} u_{1}^{n+1}=\boldsymbol{\nabla} u_{2}^{n} \cdot \mathbf{n}_{1}+z^{n \bmod (m)} u_{2}^{n}
$$

and similarly for $u_{2}^{n+1}$ on $\Gamma=\{0\} \times \mathbf{R}$ for $m=2^{l}$ and appropriate chosen $z^{0}, \ldots, z^{m-1}$. For $l=0$, Gander and Golub [2002] obtain the following contraction rate

$$
\rho(s, z)=\left(\frac{\sqrt{c+s^{2}}-z^{0}}{\sqrt{c+s^{2}}+z^{0}}\right)^{2}
$$

for the $s$-th Fourier mode. For the mesh parameter $h$, an optimization over $S=\left(s_{\text {min }}, \pi / h\right)$ gives $\min _{z^{0} \geq 0}\left(\max _{s \in S} \rho\left(s, z^{0}\right)\right)=1-\mathcal{O}(\sqrt{h})$. In the cyclic case $m=2^{l}$ one gets the rate

$$
\rho(m, s, z)=\prod_{j=1}^{m}\left(\frac{\sqrt{c+s^{2}}-z^{n \bmod (m)}}{\sqrt{c+s^{2}}-z^{n \bmod (m)}}\right)^{2 / m}
$$

and the optimized result

$$
\min _{z \geq 0}\left(\max _{s \in S} \rho(m, s, z)\right) \approx 1-\frac{4}{m}\left[\frac{\left(c+s_{\min }^{2}\right) h^{2}}{16 \pi^{2}}\right]^{\frac{1}{4 m}}, \quad h \rightarrow+0 .
$$

This result is useful for meshes with $\frac{\left(c+s_{\min }^{2}\right) h^{2}}{16 \pi^{2}} \leq 1$, but this estimate deteriorates in the singularly perturbed case, i.e. for fixed $h$ and $c \rightarrow+\infty$.

For the non-symmetric case, the optimization of the Schwarz method corresponding to $l=0$ can be found in Nataf [2001]. The extension to a cyclic Schwarz method with $l \geq 1$ is open.

\section{A posteriori based design of the interface condition}

As an alternative to the a priori based design of the interface parameter we propose an approach based on an a posteriori estimate. Consider a simplified situation with $\bar{\Omega}=\bar{\Omega}_{1} \cup \bar{\Omega}_{2} \subset \mathbf{R}^{2}$ with meas $\left(\partial \Omega \cap \partial \Omega_{i}\right)>0$ and straight interface $\Gamma=\partial \Omega_{1} \cap \partial \Omega_{2}$ of size $H=$ meas $(\Gamma) \sim \operatorname{diam}\left(\Omega_{i}\right), i=1$, 2. Set $W=H_{00}^{\frac{1}{2}}(\Gamma)$. We assume constant data $\epsilon, \mathbf{b}, c$. In Lube et al. [2000] we proved

Theorem 1. Let $u_{h}$ be the solution of (6). The DDM-subdomain error $e_{h, k}^{n}=$ $u_{h, k}^{n}-\left.u_{h}\right|_{\Omega_{k}}, k \in\{1,2\}$, can be controlled via (computable) interface data:

$$
\left.\left\|e_{h, k}^{n+1}\right\|\right|_{\Omega_{k}} \leq A_{j}\left\|u_{h, k}^{n}-u_{h, j}^{n+1}\right\|_{W}+B_{j}\left|z_{k}-\frac{\mathbf{b} \cdot \mathbf{n}_{k}}{2}\right|\left\|u_{h, k}^{n}-u_{h, j}^{n+1}\right\|_{L^{2}(\Gamma)}
$$


for $j=3-k$ and with data-dependent constants

$$
A_{j}=\sqrt{\epsilon}\left(1+\sqrt{\frac{c}{\epsilon}} H+\min \left[\frac{\|\mathbf{b}\|_{\infty} H}{\epsilon} ; \frac{\|\mathbf{b}\|_{\infty}}{\sqrt{c \epsilon}}\right]\right), \quad B_{j}=\sqrt{\frac{H}{\epsilon}} .
$$

This result motivates to equilibrate the two right-hand side terms in (11) in order to obtain information about the design of the interface parameter $z_{k}$. In Lube et al. [2000] we considered the estimate

$$
\left\|\left|e_{h, k}^{n+1}\left\|\left.\right|_{s, \Omega_{k}} \leq \max \left(A_{j} ; B_{j} C \sqrt{H}\left|z_{k}-\frac{1}{2} \mathbf{b} \cdot \mathbf{n}_{k}\right|\right)\right\| u_{h, k}^{n}-u_{h, j}^{n+1} \|_{W}\right.\right.
$$

using the continuous embedding result $\|\phi\|_{L^{2}(\Gamma)} \leq C \sqrt{H}\|\phi\|_{W}$ for all $\phi \in W$. On the other hand, an inverse estimate in (11) leads to

$$
\left\|\left|e_{h, k}^{n+1}\left\|\left.\right|_{s, \Omega_{k}} \leq \max \left(C A_{j} h^{-\frac{1}{2}} ; B_{j}\left|z_{k}-\frac{1}{2} \mathbf{b} \cdot \mathbf{n}_{k}\right|\right)\right\| u_{h, k}^{n}-u_{h, j}^{n+1} \|_{L^{2}(\Gamma)} .\right.\right.
$$

In the symmetric case $\mathbf{b}=\mathbf{0}$ we get from (13) and (14)

$$
z_{k} \sim \frac{\epsilon}{H} \sqrt{\frac{H}{L}}\left(1+H \sqrt{\frac{c}{\epsilon}}\right), \quad L \in\{h, H\} .
$$

In the non-symmetric case $\mathbf{b} \neq \mathbf{0}$, the design of $z_{k}$ has to match the hyperbolic limit of the Robin condition, i.e.

$$
0=\lim _{\epsilon \rightarrow 0} \Phi_{k}(u)=\left(-\frac{1}{2} \mathbf{b} \cdot \mathbf{n}_{k}+\lim _{\epsilon \rightarrow 0} z_{k}\right) u \text { if } \mathbf{b} \cdot \mathbf{n}_{k} \geq 0 .
$$

By extending this condition to the inflow part of $\partial \Omega_{k}$ with $\mathbf{b} \cdot \mathbf{n}_{k}<0$, we obtain from (13)-(14) as a reasonable choice

$$
\begin{aligned}
z_{k} & =\frac{1}{2}\left|\mathbf{b} \cdot \mathbf{n}_{k}\right|+R_{k}(L), \quad L \in\{h, H\} \\
R_{k}(L) & \sim \frac{\epsilon}{H} \sqrt{\frac{H}{L}}\left(1+H \sqrt{\frac{c}{\epsilon}}+\min \left[\frac{H\|\mathbf{b}\|}{\epsilon} ; \frac{\|\mathbf{b}\|}{\sqrt{c \epsilon}}\right]\right) .
\end{aligned}
$$

Inserting (16), (17) with $L=H$ in (13) and applying an inverse inequality, we obtain the optimized a posteriori estimates

$$
\left\|\left|e_{h, k}^{n+1}\left\|\left.\right|_{\Omega_{k}} \leq A_{j}\right\| u_{h, k}^{n}-u_{h, j}^{n+1}\left\|_{W} \leq C A_{j} h^{-\frac{1}{2}}\right\| u_{h, k}^{n}-u_{h, j}^{n+1} \|_{L^{2}(\Gamma)} .\right.\right.
$$

The last estimate also follows directly by inserting (16), (17) with $L=h$ in (14). Therefore we propose to extend the condition (16) to $L \in[h, H]$.

In Lube et al. [2000] we considered the case $L=H$. This choice usually allows a fast error reduction down to the discretization error level if the solution has no highly oscillatory behaviour. Fortunately, the latter case is rare for problem (1)-(2) with $c-\frac{1}{2} \nabla \cdot \mathbf{b} \geq 0$. 

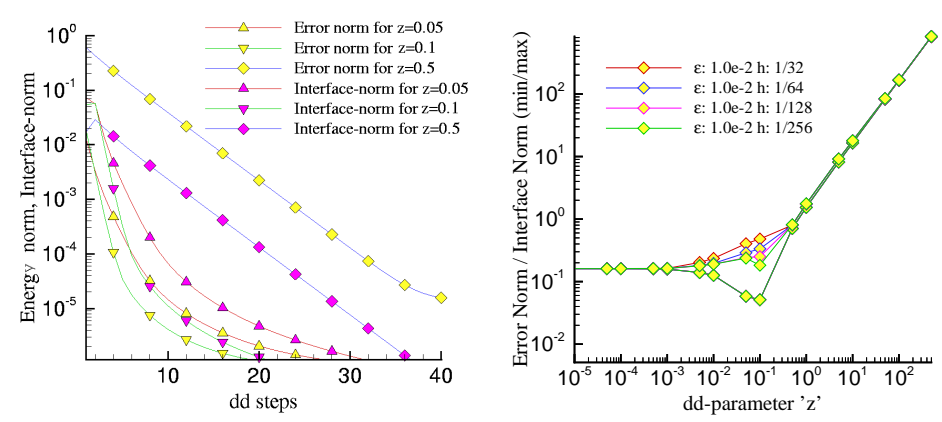

Fig. 1. Reliability of the a posteriori estimate for $h=\frac{1}{128}$ (left), Control of $\max _{n} / \min _{n} \sum_{i=1}^{4}\left\|\left|e_{h, k}^{n+1}\left\|\left.\right|_{\Omega_{i}} / \sum_{i \neq j}\right\| u_{h, i}^{n+1}-u_{h, j}^{n} \|_{L^{2}\left(\Gamma_{i j}\right)}\right.\right.$ vs. $z$ (right).

Example 1. Consider the problem (1)-(2) with $\mathbf{b} \equiv 0, \epsilon=10^{-2}, c=1$ in $\Omega=(0,1)^{2}$. The exact (smooth) solution is $u=x_{1}\left(1-x_{1}\right) x_{2}\left(1-x_{2}\right) e^{x_{1} x_{2}}$. We denote the solution of (6) with $\mathcal{P}_{1}$-elements and $h=\frac{1}{128}$ by $u_{k}=\left.u_{h}\right|_{\Omega_{k}}$. The DDM on an equidistant $2 \times 2$ macro partition with an initial guess $\Lambda_{j k}^{0}=0$ leads to the sequence $u_{h, k}^{n}$. The stopping criterion $\left.\sum_{k}\left\|\left|u_{h, k}^{n}-u_{h}\right|_{\Omega_{k}}\right\|\right|_{\Omega_{k}} \leq$ $10^{-6}$ has a tolerance beyond the discretization error level.

Fig. 1 (left) shows that the subdomain error $\left\||\cdot \||_{\Omega_{k}}\right.$ is clearly controlled by the $L^{2}(\Gamma)$ interface error according to Theorem 1. Moreover, the convergence of the DD-iteration depends strongly on $z$. The fast error reduction in the first phase corresponds to a fast reduction of "low" frequencies; but then a (very) slow reduction of "higher" modes can be seen. In Fig. 1 (right) we control the maximal/minimal (w.r.t. to the number $n$ of DD steps) ratio between the subdomain and interface errors for varying $h$. The value $z_{k} \sim \frac{1}{10}$ corresponding to the minimum of this ratio for $h=\frac{1}{256}$ is in agreement with the value predicted by (15) with $L=H$. As predicted by Theorem 1 , we observe a linear dependence of the error on $z$ for increasing $z$.

Obviously, the results of Example 1 with the optimized value of $z$ according to (16), (17) with $L=H$ depend only on the data of the problem (1)-(2) and not on $h$. We want to check this result for other typical cases.

Example 2. Let be $\Omega$ and the solution $u$ as in Example 1. The FEM solution $u_{h}$ of $(6)$ is computed with $\mathcal{P}_{1}$-elements on a fine mesh with $h=\frac{1}{256}$ and with SUPG stabilization in advection-dominated cases for
A: Symmetric case: $\quad \mathbf{b}=(0,0), \quad c=1, \quad$ DDM with 4 subdomains,
B: Case $\left|\mathbf{b} \cdot \mathbf{n}_{i}\right|>0: \quad \mathbf{b}=(2,1), \quad c=1, \quad$ DDM with 2 subdomains,
C: Case $\left|\mathbf{b} \cdot \mathbf{n}_{i}\right| \equiv 0: \quad \mathbf{b}=(0,1), \quad c=1, \quad$ DDM with 2 subdomains .

The initial guess for the Lagrange multipliers is $\Lambda_{i j}^{0}=0$. The stopping criterion for the error between the discrete solutions with and without DDM is $\sum_{k}\left\|u_{h, k}^{n}-\left.u_{h}\right|_{\Omega_{k}}\right\|_{L^{2}\left(\Omega_{k}\right)} \leq 10^{-6}$. The convergence in this range is predicted 

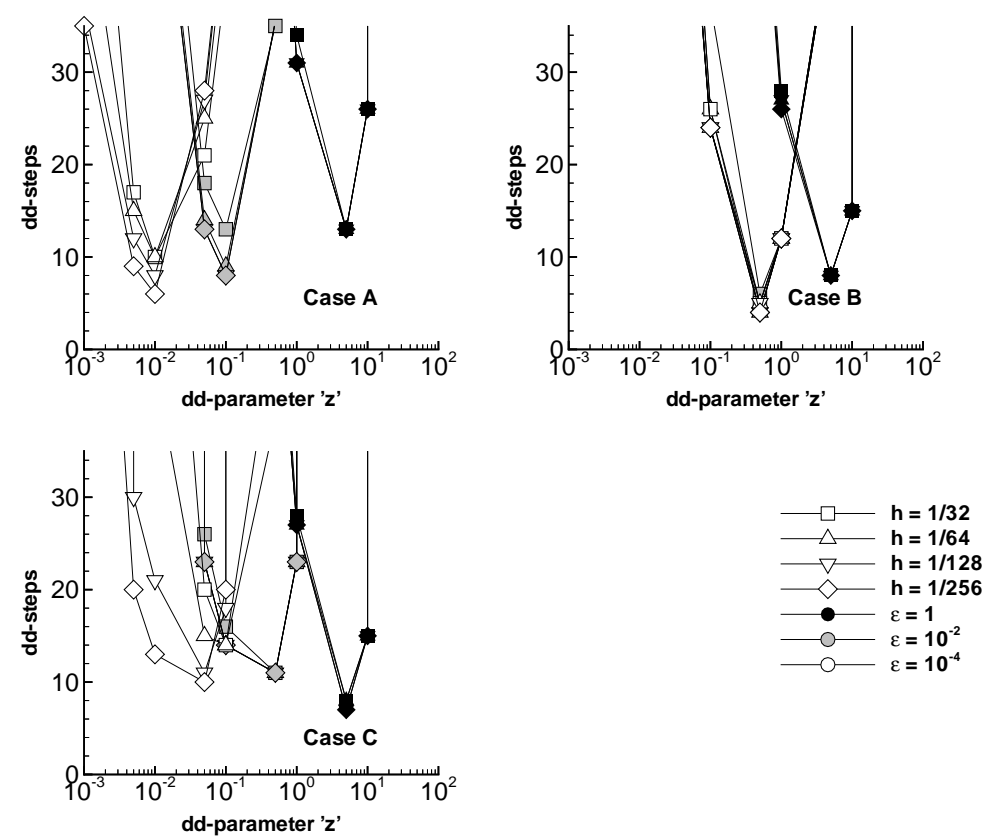

Fig. 2. Optimization of the interface parameter $z$ with one-level approach.

by the data of (1)-(2) and is $h$-independent. The optimal values of $z_{k}$ are predicted by the optimized $z_{k}$ from (16), (17) with $L=H$, see Fig. 2.

The nice convergence behaviour can be explained by the smoothness of the solutions and of the initial guess $\Lambda_{i j}^{0}$. Moreover, in our experiments we never found problems for singularly perturbed problems with sharp layers.

Nevertheless, the convergence behaviour of the Robin-DDM is not satisfactory beyond the discretization error level. Moreover, regarding our application to flow problems (Knopp et al. [2002]), in the turbulent case the solution usually has high-frequent components which may not be efficiently damped in our previous approach. As a remedy we propose a combination of the a posteriori control of the interface error with a cyclic multi-level version of the DDM:

Step 1: (optionally) Apply (7)-(8) with the optimized $z_{k}$ from (16)-(17) with $L=H$ until reduction of the interface error down to discretization error level, e.g. $\left\|u_{h, i}^{n}-u_{h, j}^{n+1}\right\|_{L^{2}(\Gamma)} \leq \kappa h^{l+1 / 2}$ for $\mathcal{P}_{l}$ elements.

Step 2: Apply (7)-(8) in a cyclic way with $p$ levels (see below) using (16)-(17) with $z_{k}^{1}, \ldots, z_{k}^{p}$ related to $L=H\left(\right.$ for $\left.z_{k}^{1}\right)$ and $l=h$ (for $z_{k}^{p}$ ), resp., and an even number (to our experience, 4 or 6 are sufficient) of DD steps per level until $\left\|u_{h, i}^{n}-u_{h, j}^{n+1}\right\|_{L^{2}(\Gamma)} \leq T O L$.

Let us discuss this approach for some cases of Example 2. First of all, we have to fix the number $p$ of levels. Assume a dyadic representation of the coarse 
and of the fine mesh of the domain $\Omega=(0,1)^{2}$ with $H=2^{-s}, h=2^{-t}, s, t \in$ N. From (16)-(17) we obtain $R_{k}(L) \leq R_{k}(h) \sim \sqrt{H / h}$, i.e. a mild dependence on $\sqrt{H / h}$. We propose the following rule: For $2^{p}<\sqrt{H / h} \leq 2^{p+1}$, take $p$ levels. Thus we obtain for a very fine mesh width $h=2^{-10}$ a number of two levels for a coarse grid width $H=2^{-5}$ and of four levels for $H=2^{-1}$.

Example 3. Consider the situation of Example 2 with a $2 \times 2$ macro partition with $H=\frac{1}{2}$ and a fine mesh with $h=2^{-6}$. This leads to $p=2$ levels. We start with the symmetric case of (1)-(2) with $\epsilon=1, \mathbf{b}=\mathbf{0}, c=1$. The fast error reduction within the first steps is followed by a very slow reduction in the one-level case, cf. Fig. 3 (left). Here $u^{h}$ and $u_{\text {seq }}^{h}$ denote the solutions with and without DDM. The two-level method with 6 DD-steps per level leads to a dramatic acceleration, cf. Fig. 3 (right).
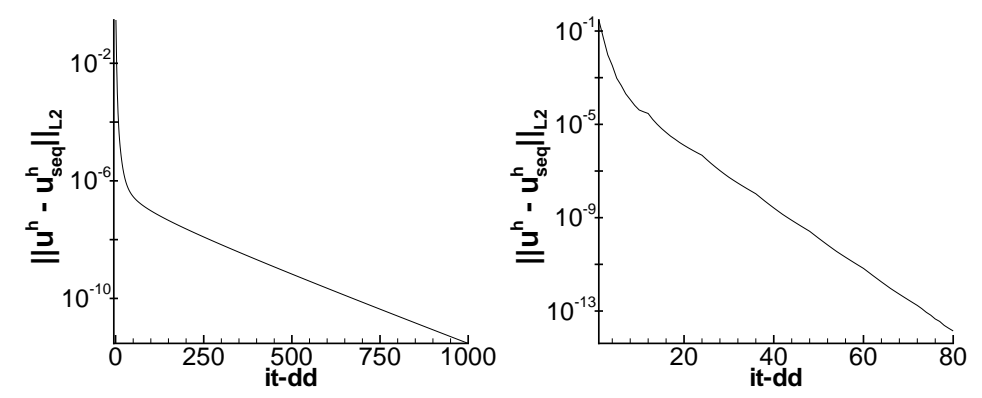

Fig. 3. Error reduction for the symmetric case: $p=1$ (left), $p=2$ (right)

Consider now the non-symmetric and advection-dominated case with $\epsilon=$ $10^{-5}, \mathbf{b}=\frac{(1,2)^{T}}{\sqrt{5}}, c=0$. In Fig. 4 we observe a similar behaviour of the proposed approach with $p=1$ (left) and $p=2$ (right) levels, although the acceleration is not so dramatic as in the symmetric case.

Finally, let us note an observation of Gander and Golub [2002] for the symmetric case: The quality of the cyclic DDM (3)-(5) with an optimized condition (10) as a solver increases with the number of levels such that no improvement can be found with Krylov acceleration. A similar behaviour is very likely in the non-symmetric case.

\section{Summary}

Considerable progress has been reached for Schwarz methods with (a priori) optimized transmission conditions. We propose an approach based on a refined a posteriori error estimate for a DDM with transmission conditions of Robin type. For the one-level variant, this condition can be optimized in such a 

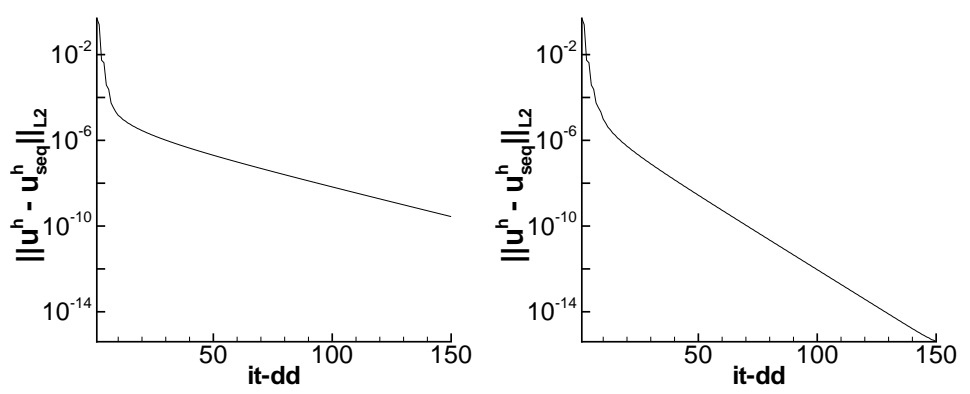

Fig. 4. Error reduction for the non-symmetric case: $p=1$ (left), $p=2$ (right)

way that the convergence is very reasonable down to the discretization error level; but then one observes a rapid slow-down of error reduction for higher error modes. This is valid for "smooth" solutions and is in contrast to highly oscillatory solutions typically appearing, e.g., for turbulent flows.

A multilevel-type method with optimized interface parameters allows a strong acceleration of the convergence. The approach is motivated by theoretical results, but more efforts are necessary to improve its present state. An advantage of the method over a priori optimized methods is the control of the convergence within the iteration. Moreover, a combination with adaptive mesh refinement is possible. It remains open whether the method is linearly convergent. Moreover, a genuinely multilevel-type implementation might be possible. Finally, the extension to incompressible flows has to be done.

\section{References}

M. J. Gander and G. Golub. A non-overlapping optimized Schwarz method which converges with arbitrarily weak dependence on $h$. In I. H. et.al., editor, Proc. Fourteenth Intern. Conf. on Domain Decomposition Methods, pages 281-288. DDM.org, 2002.

T. Knopp, G. Lube, R. Gritzki, and M. Rösler. Iterative substructuring techniques for incompressible nonisothermal flows and its application to indoor air flow simulation. Intern. J. Numer. Meths. Fluids, 40:1527-1538, 2002.

P. L. Lions. On the Schwarz alternating method III: A variant for nonoverlapping domains. In Chan et al., editor, Proc. Third Intern. Symp. on Domain Decomposition Methods, pages 202-223. SIAM, 1990.

G. Lube, L. Müller, and F. Otto. A non-overlapping domain decomposition method for the advection-diffusion problem. Computing, 64:49-68, 2000.

F. Nataf. Interface connections in domain decomposition methods. In NATO Advanced Study Institute, Modern Methods in Scientific Computing and Applications. NATO Science Ser.II., vol. 75, 2001. 